University of Nebraska - Lincoln

DigitalCommons@University of Nebraska - Lincoln

Faculty Publications, Department of Physics and Astronomy

October 2003

\title{
Elucidating the mechanisms of double ionization using intense half-cycle, single-cycle, and double half-cycle pulses
}

\author{
G. Lagmago Kamta \\ University of Nebraska-Lincoln \\ Anthony F. Starace \\ University of Nebraska-Lincoln, astarace1@unl.edu
}

Follow this and additional works at: https://digitalcommons.unl.edu/physicsfacpub

Part of the Physics Commons

Kamta, G. Lagmago and Starace, Anthony F., "Elucidating the mechanisms of double ionization using intense half-cycle, single-cycle, and double half-cycle pulses" (2003). Faculty Publications, Department of Physics and Astronomy. 45.

https://digitalcommons.unl.edu/physicsfacpub/45

This Article is brought to you for free and open access by the Research Papers in Physics and Astronomy at DigitalCommons@University of Nebraska - Lincoln. It has been accepted for inclusion in Faculty Publications, Department of Physics and Astronomy by an authorized administrator of DigitalCommons@University of Nebraska Lincoln. 


\title{
Elucidating the mechanisms of double ionization using intense half-cycle, single-cycle, and double half-cycle pulses
}

\author{
G. Lagmago Kamta* and Anthony F. Starace \\ Department of Physics and Astronomy, The University of Nebraska, 116 Brace Laboratory, Lincoln, Nebraska 68588-0111, USA
}

(Received 4 February 2003; published 27 October 2003)

\begin{abstract}
We investigate the interaction of a two-active electron system $\left(\mathrm{Li}^{-}\right)$with intense single-cycle and double half-cycle pulses. The "intensity" and "frequency" considered correspond to the "multiphoton above-barrier regime." For the single-cycle pulse (SCP), the electric field changes sign once, allowing electron wave packets created during the first half cycle to recollide with the parent ion when driven back by the field. For the double half-cycle pulse (DHP), however, the electric field does not change sign, and electron wave packets created during the first half cycle are not driven back to the parent ion. We find that both single and double ionization are significantly larger for the SCP than for the DHP, thereby elucidating the role of the rescattering mechanism. On the other hand, doubly ionized electrons produced by a half-cycle pulse and a DHP are found to have angular distributions in which one electron is ejected in the direction of the pulse field, and the other in the opposite direction. This clear signature of electron correlations suggests that "shake-off," "knockout," and, possibly, "multiphoton-sharing" processes are alternative contributing mechanisms for double ionization in this regime.
\end{abstract}

DOI: 10.1103/PhysRevA.68.043413

PACS number(s): 32.80.Rm, 32.80.Gc, 32.80.Wr, 31.70.Hq

\section{INTRODUCTION}

There has been a great deal of interest in understanding the process of double ionization by single and multiple photon impact. For the weak field, single photon regime [1], various mechanisms have been investigated (see, e.g., Refs. [2-4] and references therein). In the shake-off mechanism, one electron is ejected so rapidly after absorbing the photon that the other is shaken out, since it cannot adiabatically adjust to the new ionic potential; screening of the nucleus by the second electron plays a crucial role. In the knockout mechanism, one electron absorbs the photon, but as this electron exits the atom, it undergoes a hard binary collision with the other electron that ionizes it; here electron correlation in the final state plays the crucial role. In the photon-sharing mechanism, the two electrons share the photon energy and are ionized with almost equal but opposite momenta; correlation in the initial bound state plays the crucial role here [2]. In general, of course, these various mechanisms interfere; only for very specific situations is it possible to physically distinguish them.

Attempts to establish parallels to these mechanisms for high intensity, multiphoton ionization by intense laser fields have been prompted by the observation of nonsequential double ionization $[5,6]$, as well as by more recent differential measurements of the recoil momentum distribution $[7,8]$. Shake-off [5], rescattering [9], and collective tunneling [10] have been suggested as potential mechanisms for intense field double ionization. For the collective tunneling mechanism, both electrons tunnel together through the Coulomb potential barrier that is lowered by the intense laser field. In

\footnotetext{
*Present address: Laboratoire de Chimie Théorique, Faculté des Sciences, Université de Sherbrooke, Sherbrooke, Canada QC J1K2R1.
}

the rescattering mechanism, one electron is initially set free by tunneling, then accelerated in the laser field, and later driven back to collide with its parent ion. In this collision, the second electron may be knocked free or excited, with excitation eventually converted into ionization by the laser field.

In order to approximately distinguish tunneling ionization from conventional multiphoton ionization, the Keldysh adiabaticity parameter $\gamma \equiv \sqrt{I_{p} / 2 U_{p}}$ is often used, where $I_{p}$ is the ionization potential of the target system, and $U_{p}$ is the ponderomotive potential. Tunneling is favored over multiphoton ionization for $\gamma \ll 1$. Most experiments on double ionization by ultrashort intense laser fields have been done for frequencies and intensities corresponding to the tunneling regime [5-8]. A rigorous theoretical investigation of these processes requires a direct numerical integration of the fulldimensional, time-dependent Schrödinger equation (TDSE) describing the atomic system in interaction with the laser pulse. For two-electron systems, such theoretical approaches are available and are based on angular-momentum expansions of the wave function [11-13]. However, they have not been used for calculations in the tunneling regime, because of the large basis expansion necessary for the wave function, which in turn requires huge computer resources for the computation. However, many simpler, approximate methods, based on classical $[7,14]$ and semiclassical approaches $[15,16]$, on one- and two-dimensional model atoms $[17,18]$, on $S$-matrix approaches $[19,20]$, etc., find that rescattering is the primary mechanism for double ionization in the tunneling regime.

The recent observation of self-amplified spontaneous emission at extreme ultraviolet wavelengths in a freeelectron laser [21] opens the route to experimental studies of laser-atom interactions for much higher laser frequencies (e.g., from the vuv to the x-ray ultraviolet regime) than are currently used. These higher frequencies correspond to the 
multiphoton regime in He. This breakthrough opens a new domain of laser-atom interactions, in which ab initio twoelectron calculations are computationally feasible and in which experimental data will be available for comparison. In the multiphoton regime (for which $\gamma>1$ ), fewer photons are necessary to double ionize as compared to the tunneling regime, so that $a b$ initio full-dimensional, two-electron calculations are tractable. In this work, we perform such a calculation, in an attempt to probe the mechanisms for double ionization in the multiphoton above-barrier regime. We investigate the single and double ionization of a two-active electron system $\left(\mathrm{Li}^{-}\right)$by a single-cycle pulse (SCP) and by a double half-cycle pulse (DHP). For the DHP, the electric field is oriented in the same direction during both half cycles, so that a wave packet created during the first half cycle is further driven away from the core by the second half cycle. For the SCP, the electric-field direction during the first half cycle is opposite to that of the next half cycle (as is usual for a light wave), thereby allowing a wave packet created during the first half cycle to be driven back so that it recollides with the parent ion during the second half cycle. Our results show that both single and double ionization are enhanced for the SCP case as compared to the DHP case, thereby suggesting a strong role for rescattering in these processes. We find also that angular distributions for double ionization show evidence of the strong influence of electron-electron and electron-core effects. In particular, the angular distributions for double ionization evaluated after a half-cycle pulse (HCP) suggest the existence of entangled contributions from other mechanisms besides rescattering, such as shake-off, knockout, and, possibly, multiphoton-sharing mechanisms.

Note that the rescattering mechanism has been considered up to now mostly in the tunneling regime, in which the first electron appears in the continuum with zero initial velocity after tunneling. The corresponding classical kinematics leading to a recollision with the parent ion have been investigated extensively [22]. Our two-electron calculations correspond to the multiphoton, above-barrier regime, so that the first electron appears in the continuum with a nonzero velocity. For this case, we perform a classical study of the kinematics of such a free electron and show that a recollision is indeed possible under specific conditions.

In our consideration of single cycle and double half-cycle pulses, we are performing numerical "experiments" in order to uncover the underlying physics of double ionization induced by an intense laser pulse. However, it is worth noting that very short pulses down to the single-cycle level are becoming possible in real experiments. In fact, recent technologies for ultrashort pulse generation have pushed the duration of pulses close to the single-cycle limit $[23,24]$. Moreover, experimentalists have also developed means to produce halfcycle pulses [25]. We note finally that we have treated elsewhere $[12,13]$ the case of short but many cycle pulses; the current work elucidates further those results.

This paper is organized as follows: In Sec. II, we investigate classically the conditions under which an electron initially free in a laser field with a nonzero velocity undergoes a recollision with the parent ion. We describe our numerical approach to solving the TDSE in Sec. III. Results for single and double ionization are presented in Sec. IV for both the SCP and the DHP. In Sec. V, we compare and discuss angular distributions for two-electron ionization by a HCP, a SCP, and a DHP. Our conclusions are given in Sec. VI. Unless specified otherwise, atomic units (a.u.) are used throughout this paper, and all angles are given in radians.

\section{CLASSICAL RESCATTERING MODEL WITH INITIAL VELOCITY}

In this section, we consider a one-electron classical model: an electron is at a given time $t_{0}$ "born" in the continuum in the field of the binding potential of the parent ion with initial velocity $\mathbf{v}_{0}$ at the coordinate $z_{0}$ (which we choose to be the origin, i.e., $z_{0}=0$ ). The subsequent motion of the electron is treated classically, neglecting the binding potential. We are interested in finding how the initial time $t_{0}$, the initial velocity $\mathbf{v}_{0}$, and the laser field parameters influence the return to the origin of the electron for a recollision with the parent ion. Models of this type are well known as the "simpleman's theory" or the "quasiclassical model" [9,22], and are often considered for the tunneling regime, in which the electron is born in the continuum with zero initial velocity.

Consider an electron in the laser field characterized by the electric field

$$
\mathbf{E}(t)=\hat{\mathbf{z}} E_{0} \sin \omega t,
$$

where $\hat{\mathbf{z}}$ is the unit vector along the polarization axis, $E_{0}$ is the electric-field amplitude, and $\omega$ the frequency. Solving the classical equation of motion of the electron, $m \ddot{z}$ $=-e E_{0} \sin \omega t$, with initial conditions $\dot{z}\left(t_{0}\right)=v_{0}$ and $z\left(t_{0}\right)$ $=z_{0}$, gives for $t>t_{0}$ the electron momentum

$$
\mathbf{p}\left(t, t_{0}\right)=\frac{e E_{0}}{\omega}\left(\cos \omega t-\cos \omega t_{0}\right) \hat{\mathbf{z}}+\mathbf{p}_{0},
$$

and the electron position

$$
z(t)=\frac{e E_{0}}{m \omega^{2}}\left[\sin \omega t-\sin \omega t_{0}+\left(\eta \beta-\cos \omega t_{0}\right)\left(\omega t-\omega t_{0}\right)\right],
$$

where $\mathbf{p}_{0}=m \mathbf{v}_{0}=\eta m v_{0} \hat{\mathbf{z}}$ is the initial momentum of the electron at its birth. The quantity $\eta= \pm 1$ specifies the direction of the initial velocity $\mathbf{v}_{0}$ with respect to the laser polarization axis: $\eta=+1$ for $\mathbf{v}_{0}$ along positive $\hat{\mathbf{z}}$, and $\eta=-1$ for $\mathbf{v}_{0}$ along negative $\hat{\mathbf{z}}$. The quantity $\beta$ is given by

$$
\beta=\sqrt{\frac{K_{0}}{2 U_{p}}},
$$

where $K_{0}=m v_{0}^{2} / 2$ is the initial kinetic energy of the electron, and

$$
U_{p}=\frac{\left(e E_{0}\right)^{2}}{4 m \omega^{2}}
$$


is the ponderomotive potential. The parameter $\beta$ depends on the ratio of the kinetic energy of the electron at its birth to the ponderomotive potential energy. It is clear that $\beta$ is very similar to, but different from, the Keldysh parameter $\gamma$ $=\sqrt{I_{p} / 2 U_{p}}$, which involves the ratio of the ionization potential energy $I_{p}$ for the active electron to the ponderomotive potential energy.

The electron returns to the origin at times $t_{1}=t_{1}\left(t_{0}, \beta\right)$ given by

$$
\sin \omega t_{1}-\sin \omega t_{0}=\left(\cos \omega t_{0}-\eta \beta\right)\left(\omega t_{1}-\omega t_{0}\right) .
$$

Unlike the case of an electron born with zero initial velocity [22], for which the return time of the electron depends only on its birth time, the return time of the electron in the present case depends also on the parameter $\beta$. For a laser field described by Eq. (1), the electric force driving electrons is oriented along negative $\hat{\mathbf{z}}$ for $2 n \pi<\omega t<(2 n+1) \pi$, and along positive $\hat{\mathbf{z}}$ for $(2 n+1) \pi<\omega t<(2 n+2) \pi$, where $n$ is an arbitrary positive integer. Therefore, electrons ejected at times $t$ satisfying $2 n \pi<\omega t<(2 n+1) \pi$ have velocities along negative $z$ (i.e., $\eta=-1$ ), whereas electrons free at times $t$ such that $(2 n+1) \pi<\omega t<(2 n+2) \pi$ have velocities along positive $z$ (i.e., $\eta=+1$ ). In our discussion below, we focus on electrons ejected at times $t_{0}$ such that $0<\omega t_{0}<\pi$, i.e., corresponding to the first half cycle, for which $\eta=-1$. Also, our subsequent analysis will always involve the scaled variables $\omega t_{0}$ and $\omega t_{1}$, instead of $t_{0}$ and $t_{1}$, so that our conclusions are applicable for any laser frequency.

Depending on the initial time $t_{0}$ and the parameter $\beta$, Eq. (6) has zero, one, or many solutions. Our numerical simulations indicate that there is a maximum value $\beta_{c} \approx 1.2172$ of $\beta$, such that for $\beta>\beta_{c}$, Eq. (6) has no solution at all (regardless of $t_{0}$ ), i.e., the electron is born with such a large kinetic energy that the laser field is not strong enough to stop the electron and drive it back to the origin. For $\beta \leqslant \beta_{c}$, whether or not the electron returns depends on its birth time $t_{0}$. Data on the number of electron returns with respect to $t_{0}$ are summarized in Fig. 1 for various values of the parameter $\beta$. For $\beta=0$, which corresponds to the birth of the electron with zero energy (tunneling case), the electron never returns for $0<\omega t_{0} / \pi<1 / 2$ (i.e., during the initial rise of the pulse from zero to its first maximum), returns at least twice for $1 / 2<\omega t_{0} / \pi<0.570$, and returns exactly once for 0.570 $<\omega t_{0} / \pi<1$ (this case has been studied in Ref. [22]). Thus, for $\beta=0$, only those electrons born just after the field reaches its maximum return for a possible recollision. For $\beta=0.2$, Fig. 1 shows that the electron never returns for 0 $<\omega t_{0} / \pi<0.563$, returns at least twice for $0.563<\omega t_{0} / \pi$ $<0.636$ and, returns exactly once for $0.636<\omega t_{0} / \pi<1$. In general, with increasing $\beta$, in order for the electron to return, its birth time must increase, i.e., electrons that return are born increasingly later, after the maximum of the first half cycle, and increasingly closer to $t_{0}=\pi / \omega$, the time when the first half cycle vanishes. Electrons born closer to the end of the first half cycle do not have time to be driven far enough to escape the field before the electric field changes sign and drives them to the origin.

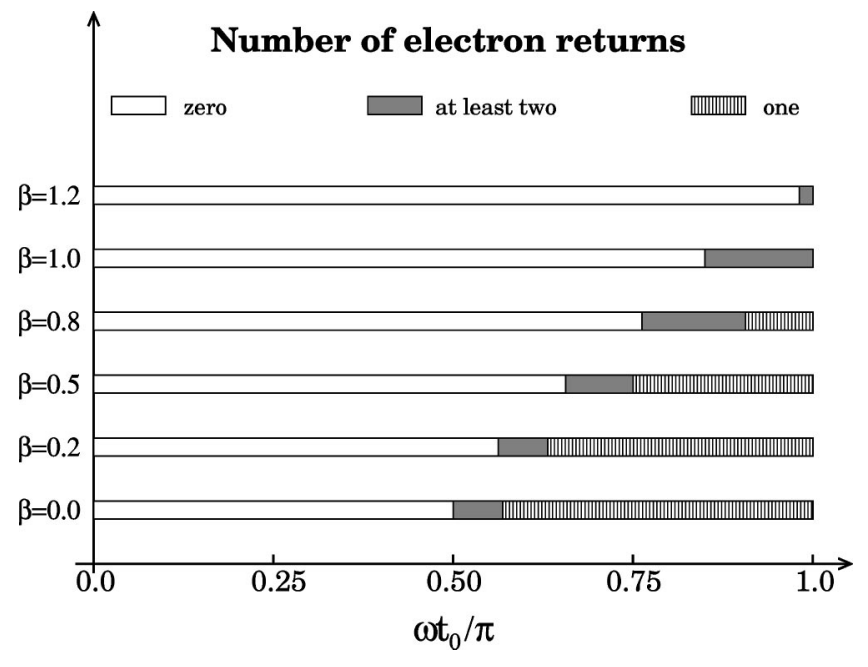

FIG. 1. Number of returns of the electron to the origin (possible rescatterings), as a function of its birth time $t_{0}$ in the laser field, for various values of the parameter $\beta=\sqrt{K_{0} / 2 U_{p}}$, where $K_{0}$ is the initial kinetic energy and $U_{p}$ is the ponderomotive potential. The white, dark, and hatched patterns indicate zero, at least two, and only one return(s) of the electron, respectively. These results are obtained by solving classical equations for an electron that becomes free at time $t_{0}$ in a laser field of frequency $\omega$ (i.e., the interaction of the electron with the atomic core is ignored).

We now focus specifically on the first return of the electron. A plot of the first return time $t_{1}$ with respect to the birth time $t_{0}$ is given in Fig. 2 for various values of $\beta$; one sees that the return time of the electron decreases with the in-

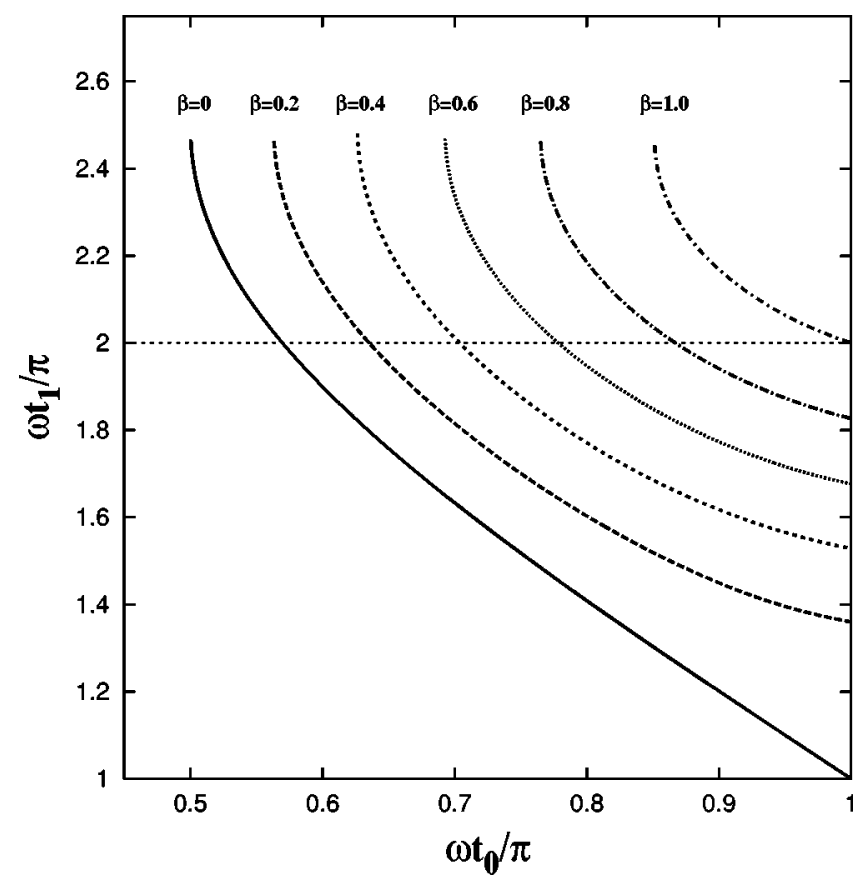

FIG. 2. First return time $t_{1}$ of the electron vs the ejection time $t_{0}$ for various values of the parameter $\beta=\sqrt{K_{0} / 2 U_{p}}$ (see text for details). The horizontal dashed line indicates the end of the first laser oscillation. These results are obtained using a classical approach, as described in Fig. 1. 
crease of its birth time. In other words, the earlier an electron is born during the first half cycle, the later it returns to the parent ion. This is due to the fact that electrons born early are accelerated by the field in the same direction as their initial velocity for a much longer time than those born later and closer to the vanishing point of the first half cycle, thereby leading to a larger return time than electrons born later. Electrons having the shortest return times are born just before the end of the first half cycle, and, depending on $\beta$, this return occurs before the middle of the second half cycle, i.e., with a delay much less that a quarter of the laser period. Figure 2 shows that for $\beta=0$, for example, electrons born just after the maximum of the field (i.e., at times $t_{0}$ such that $1 / 2$ $\left.<\omega t_{0} / \pi<0.570\right)$ return to the parent ion at a time $t_{1}$ satisfying $\omega t_{1}>2 \pi$ (i.e., after the end of the second half cycle). These electrons are accelerated back to the atomic core during the second half laser cycle, but only reach the core afterwards. On the other hand, electrons freed at $t_{0}$ such that $0.570 \leqslant \omega t_{0} / \pi<1$ return before the end of the second half cycle. For $\beta=1$ the first return of the electron occurs exactly at $t_{1}=2 \pi / \omega$, i.e., when the second half cycle vanishes, and for $1.0<\beta<\beta_{c}$ the first return occurs after the end of the second half cycle.

The average kinetic energy of the free electron in the laser field is

$$
\left\langle\frac{1}{2} m v^{2}\right\rangle=U_{p}\left[1+2\left(\cos \omega t_{0}-\eta \beta\right)^{2}\right] .
$$

Therefore, the maximum average kinetic energy of the electron in the field is $[3+2 \beta(2+\beta)] U_{p}$, which is larger than the maximum average $3 U_{p}$ obtained for the case $\beta=0$ [26]. On the other hand, the maximum kinetic energy of the electron at its first return to the core is shown in Fig. 3 for various values of the parameter $\beta$. It appears that with increasing $\beta$, the maximum kinetic energy of the electron at its first return to the parent ion decreases with increasing $\beta$, starting from the well-known value $3.17 U_{p}$ for $\beta=0$ [27]. Therefore, the strength of the recollision decreases with increasing $\beta$.

The above analysis indicates that rescattering does not occur at all for values of $\beta$ larger than $\beta_{c} \approx 1.2172$. It only occurs for $\beta<\beta_{c}$. These conclusions are derived from a classical, single active electron calculation that neglects the Coulomb attraction of the parent atom or ion. With regard to our two-active electron model for $\mathrm{Li}^{-}$, this classical calculation assumes that the second active electron perfectly screens the Coulomb field of the $\mathrm{Li}^{+}$core, and that polarization of the $\mathrm{Li}$ atom by the first electron is negligible. Including the effects of imperfect screening or of polarization would significantly alter these classical conclusions. In particular, electrons having larger values of $\beta$ would return to the parent ion. Moreover, in a quantum-mechanical picture an electron revisiting the ion even with a large impact parameter has a significant probability to undergo a collision with the ion. Also in this picture, the Coulomb potential between the electron and the ion reduces the transverse spread of the electronic wave packet, thereby enhancing the probability for the electron to revisit the ion [15]. Therefore, by including Coulomb and quantum effects, as we do in subsequent sections

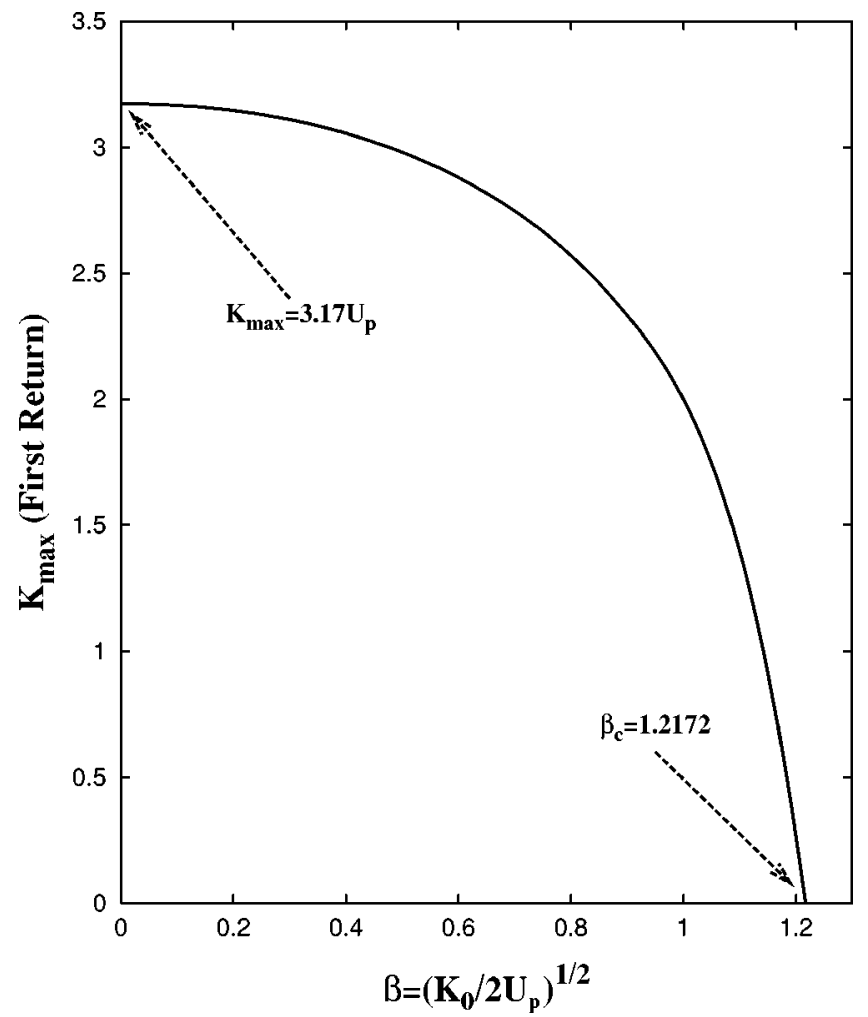

FIG. 3. Maximum kinetic energy $K_{\max }$ of the electron at its first return to the core (in units of the ponderomotive potential $U_{p}$ ) vs the parameter $\beta=\sqrt{K_{0} / 2 U_{p}}$ (see text for details). These results are obtained using a classical approach, as described in Fig. 1.

of this paper, a recollision of the electron with the parent ion occurs even for values of $\beta$ larger than the classical, freeelectron limit $\beta_{c}$.

\section{THE SOLUTION OF THE TDSE}

Since we are interested in the interaction of a two-active electron system with an ultrashort intense laser field, we need a nonperturbative, direct numerical approach for solving the TDSE, with proper account of electron correlations. The TDSE is

$$
i \frac{\partial}{\partial t} \Psi\left(\mathbf{r}_{1}, \mathbf{r}_{2}, t\right)=\left[H_{0}+D(t)\right] \Psi\left(\mathbf{r}_{1}, \mathbf{r}_{2}, t\right),
$$

where

$$
H_{0}=\frac{1}{2}\left(\mathbf{p}_{1}^{2}+\mathbf{p}_{2}^{2}\right)+V\left(r_{1}\right)+V\left(r_{2}\right)+\frac{1}{r_{12}}
$$

and where $\mathbf{p}$ and $\mathbf{r}$ denote the momentum and the coordinate of the electron, respectively, with the indices 1 and 2 referring to each of the two electrons. $r_{12}=\left|\mathbf{r}_{1}-\mathbf{r}_{2}\right|$ is the interelectronic distance and $V(r)$ is the potential that describes the interaction of each electron with the core. $D(t)$ describes the interaction of the system with the laser field. In the dipole 
approximation, $D(t)$ is given by either $D(t)=\mathbf{E}(t) \cdot\left(\mathbf{r}_{1}\right.$ $\left.+\mathbf{r}_{2}\right)$ or $D(t)=\mathbf{A}(t) \cdot\left(\mathbf{p}_{1}+\mathbf{p}_{2}\right)$ in the length gauge and velocity gauge, respectively.

We have recently developed an approach to solve Eq. (8) including all spatial dimensions for a linearly polarized laser pulse and used it to study the interaction of $\mathrm{Li}^{-}$with an intense laser field $[12,13]$. In this approach, we solve the TDSE in a spherical box using a configuration-interaction expansion of the time-dependent wave function in terms of one-electron atomic orbitals. Due to its low binding energy, nonperturbative behavior of $\mathrm{Li}^{-}$in the presence of a laser field sets in at fairly low laser intensities $\left(\approx 10^{10} \mathrm{~W} / \mathrm{cm}^{2}\right)$. This feature, in addition to the absence of a Rydberg series in the one-electron detachment spectrum of $\mathrm{Li}^{-}$, makes the time propagation of the TDSE easily tractable because it requires a reasonable box size (with a radius of about 250 a.u.). A detailed description of our numerical approach is given elsewhere $[12,13]$.

In this work, we use the length gauge for the description of the interaction of the system with the linearly polarized laser field. Our preference for the length gauge relies on the fact that it directly involves the electric field $\mathbf{E}(t)$, which unambiguously determines the direction of the electromagnetic force acting on the electrons. In addition, in this gauge, the energy operator of the system equals the unperturbed, field-free Hamiltonian $H_{0}$. This means that wave functions representing field-free eigenstates are unchanged during and after the laser-pulse excitation. Therefore, projections of the time-dependent wave function onto field-free eigenstates are gauge-invariant probability amplitudes [28-30].

We consider two "laser" pulses, linearly polarized along the $z$ axis, and having the same "frequency" and "peak intensity," but differing in shape [31]: The first pulse is given by

$$
\mathbf{E}(t)=\mathbf{E}_{S C P} \equiv\left\{\begin{array}{l}
\hat{\mathbf{z}} E_{0} \sin (\omega t), \quad 0 \leqslant t \leqslant 2 \pi / \omega \\
0 \quad \text { otherwise, }
\end{array}\right.
$$

where $\hat{\mathbf{z}}$ is the unit vector along the polarization axis. We shall refer to the pulse given by Eq. (10) as the SCP. It is plotted in Fig. 4(a), which shows that the electric field changes direction after the first half cycle, so that electron wave packets created during the first half cycle have the possibility of being driven back to the parent ion during the second half cycle. The second pulse is given by

$$
\mathbf{E}(t)=\mathbf{E}_{D H P} \equiv\left\{\begin{array}{l}
\hat{\mathbf{z}} E_{0}|\sin (\omega t)|, \quad 0 \leqslant t \leqslant 2 \pi / \omega \\
0 \quad \text { otherwise, }
\end{array}\right.
$$

which we refer to as the DHP. It is plotted in Fig. 4(b), which shows that the electric field is oriented in the same direction along the $z$ axis during both half cycles, so that throughout the pulse, the system receives two kicks in the same direction. In contrast to the DHP, the SCP allows for an enhancement of the spatial electron-electron and electron-core correlations during the second half cycle, when the electron returns to the core.
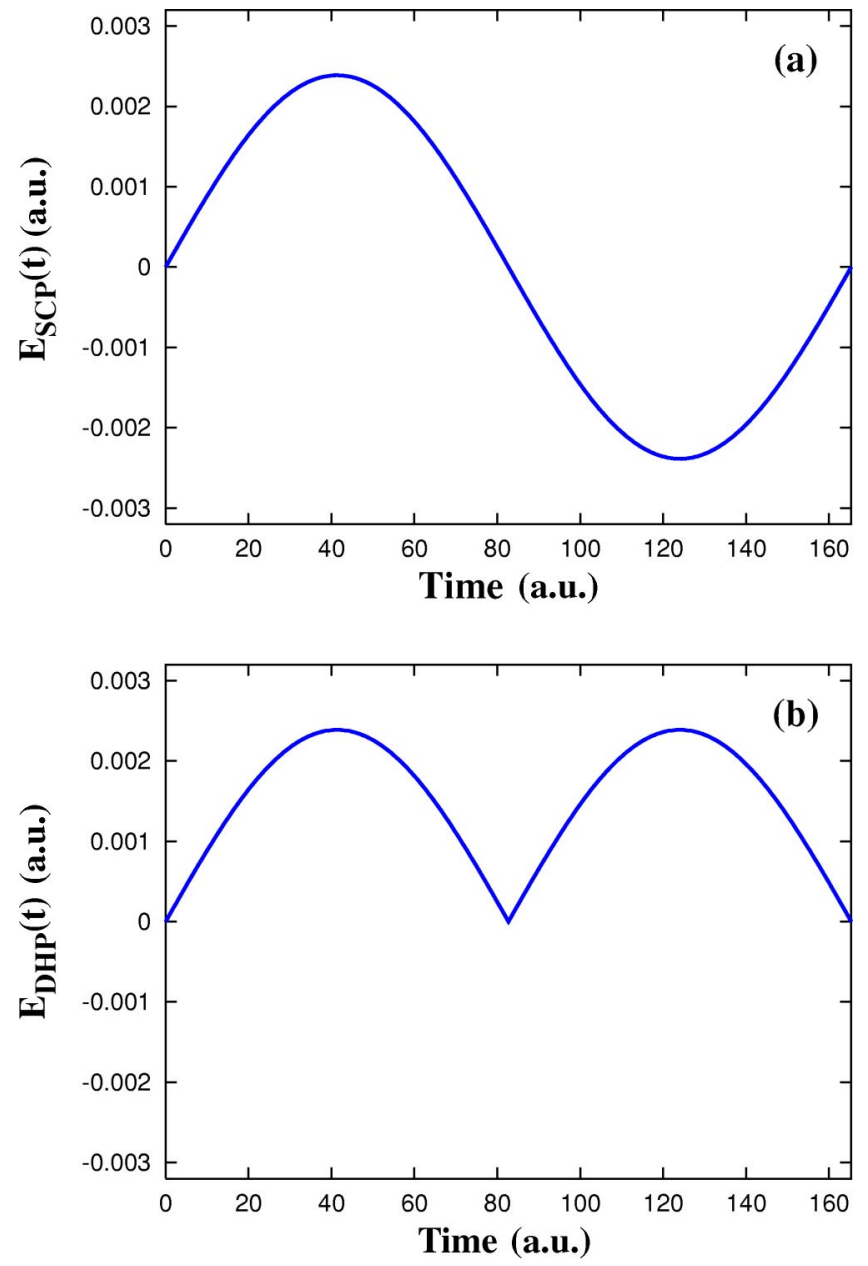

FIG. 4. Time dependence of the electric field $E(t)$, for $\omega$ $=0.038$ a.u. and $I=2 \times 10^{11} \mathrm{~W} / \mathrm{cm}^{2}$ : (a) for the single-cycle pulse (SCP) and (b) for the double half cycle pulse (DHP).

For our simulations, we consider a frequency $\omega$ $=0.038$ a.u. $(1.03 \mathrm{eV})$ and a peak intensity $I=2$ $\times 10^{11} \mathrm{~W} / \mathrm{cm}^{2}$, corresponding to a ponderomotive energy of about 0.001 a.u. The binding energy of $\mathrm{Li}^{-}$is 0.0224 a.u. $(0.61 \mathrm{eV})$, and the double ionization threshold, $\mathrm{Li}^{+}$, is at an energy 0.2205 a.u. $(6.00 \mathrm{eV})$ above the ground state (see, e.g., Fig. 2 in Ref. [13]). For the above-mentioned frequency, a single-photon absorption ejects the outer electron with energy 0.0156 a.u. $(0.42 \mathrm{eV})$, whereas an absorption of at least six photons is necessary to ionize both electrons. For the ionization potential 0.0224 a.u. of $\mathrm{Li}^{-}$, and for a pulse having the characteristics described above, the Keldysh parameter is $\gamma=3.35$, which indicates that we are in the multiphoton regime. Also, the laser frequency considered is above the single-ionization threshold of $\mathrm{Li}^{-}$, and thus indicates that we are in the above-barrier ionization regime. From the perspective of the rescattering mechanism, under these conditions the first electron does not tunnel into the continuum with zero velocity, as is the case for the tunneling regime. The first electron rather "appears" in the continuum with a nonzero velocity, after absorbing one or many photons. For the above-mentioned laser frequency and pulse peak intensity, if one assumes that the outer electron of $\mathrm{Li}^{-}$is ejected in the 
continuum after absorbing one photon, one obtains $\beta$ $=2.79$. As discussed in Sec. II, a rescattering of the electron is still possible for the case of a single-cycle pulse, even though $\beta>\beta_{c}$, owing to Coulomb, polarization, and quantum effects. Also, the very short duration (about $4 \mathrm{fs}$ ) of the SCP and DHP considered in this work corresponds to a broad frequency spectrum, which contains frequency components corresponding to values of the parameter $\beta$ that are smaller than 2.79. In subsequent sections, we present and discuss the results obtained for single and double ionization of $\mathrm{Li}^{-}$by a SCP and a DHP, following a numerical solution of the TDSE with inclusion of correlations.

\section{SINGLE AND DOUBLE IONIZATION}

If $\Psi\left(\mathbf{r}_{1}, \mathbf{r}_{2}, t\right)$ is the solution of the TDSE at time $t$, then the depletion of the ground state at time $t$ is

$$
P(t)=1-\left|\left\langle\Psi_{0}\left(\mathbf{r}_{1}, \mathbf{r}_{2}\right) \mid \Psi\left(\mathbf{r}_{1}, \mathbf{r}_{2}, t\right)\right\rangle\right|^{2},
$$

where $\Psi_{0}\left(\mathbf{r}_{1}, \mathbf{r}_{2}\right)$ is the field-free ground-state wave function (because we use the length gauge, $\left\langle\Psi_{0}\left(\mathbf{r}_{1}, \mathbf{r}_{2}\right) \mid \Psi\left(\mathbf{r}_{1}, \mathbf{r}_{2}, t\right)\right\rangle$ is indeed the gauge-invariant probability amplitude for the ground-state $[28-30])$. Since $\mathrm{Li}^{-}$has only one bound state, $P(t)$ also represents the total detachment yield, which is the sum of probabilities for single ionization, double ionization, and double excitation. The time dependence of the total detachment yield and the single-ionization probability of $\mathrm{Li}^{-}$ are shown in Figs. 5(a) and 5(b) for the cases of the SCP and the DHP, respectively. The single-ionization probability is only slightly smaller than the total detachment yield, and both have a similar pattern. This is an indication that double ionization and double excitation are much smaller than single-ionization, so that the latter is almost identical to the total detachment yield. In other words, single-ionization is the dominant process. As expected, results obtained for the two pulses are identical throughout the first half cycle. During the second half cycle, the total detachment yield and the single ionization probability increase significantly for the SCP but only slightly for the DHP case. This means that the recollision that occurs for the SCP enhances both the total detachment and the single ionization probability. Following the laser-assisted recollision that occurs for the SCP, the rescattered electron gains significant energy to escape the field, thereby enhancing single ionization.

The fact that the increase in the single-ionization probability during the second half cycle is much less for the DHP than for the SCP can be understood in terms of quantum interference between electron wave packets produced during each half cycle. For the SCP, the electron wave packet created during the second half cycle (wave packet 2) propagates in the direction opposite to that of the electron wave packet created during the first half cycle (wave packet 1), resulting in little interference between the two. (The wave packets comprise non-overlapping sets of momentum eigenstates.) In contrast, for the DHP, the corresponding wave packet 2 propagates in the same direction as wave packet 1; the two wave packets are identical in every way other than the fact that they are produced at slightly different times. Because the
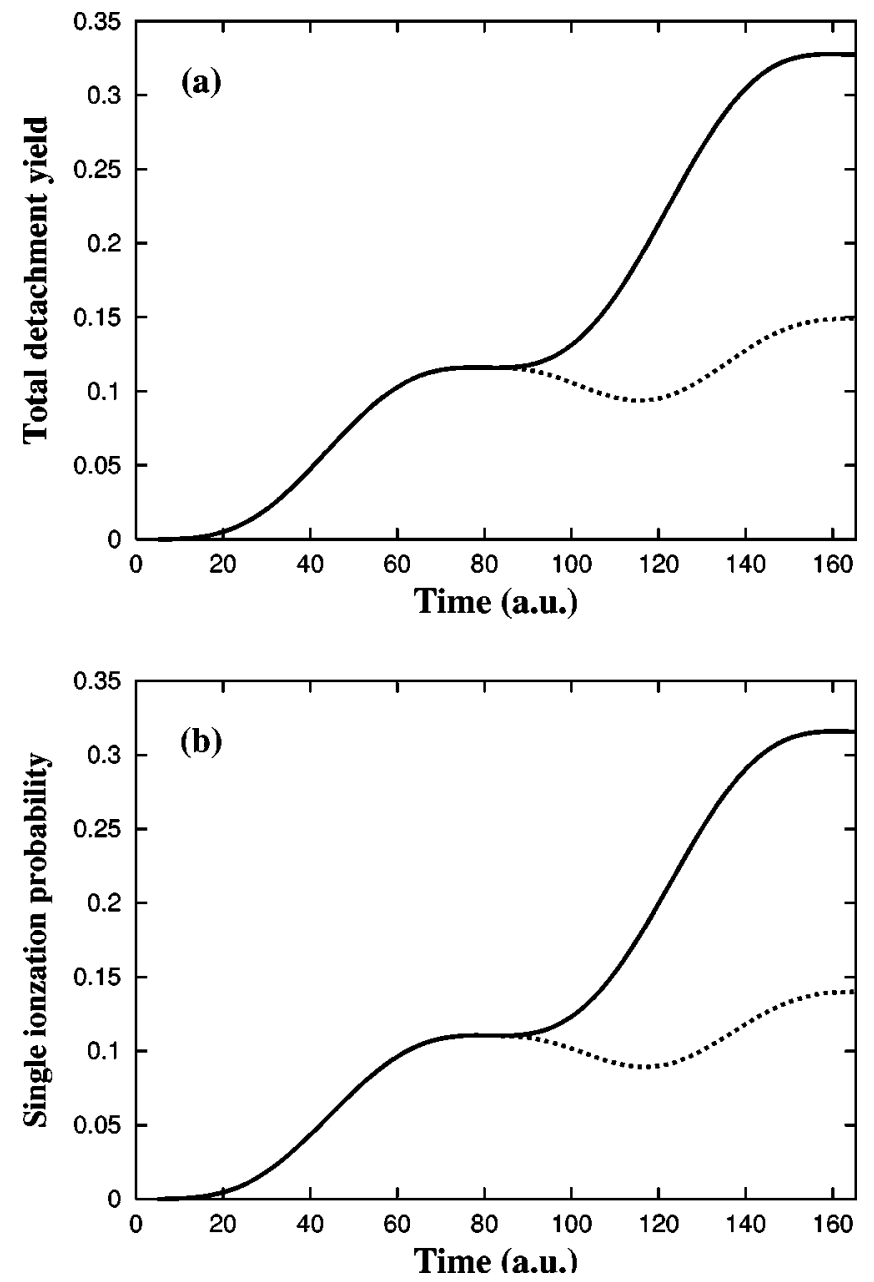

FIG. 5. Time evolution of (a) the total detachment yield and (b) the single-ionization probability for $\mathrm{Li}^{-}$, obtained using the singlecycle pulse (solid lines) and the double half cycle pulse (dashed lines). The parameters used for both pulses are intensity $I=2$ $\times 10^{11} \mathrm{~W} / \mathrm{cm}^{2}$, frequency $\omega=0.038$ a.u., and pulse duration $T$ $=2 \pi / \omega \approx 4.0 \mathrm{fs}$.

time interval separating the two wave packets is small and the spatial extent of the wave packets is large (comparable to that of the diffuse ground-state wave function from which they are produced), the wave packets interfere [32]. (When we insert a half-cycle time delay between the two half-cycle pulses, the DHP then produces roughly double the single ionization as a single HCP.) For the time interval involved in our calculations, this interference is destructive, leading to only a small increase in the single-ionization probability during the second half of the DHP. Classically, we interpret this result as due to the repulsion that the second electron wave packet "feels" when it tries to move in the same direction as the first wave packet, which suppresses the ionization of the second wave packet. The key point of Fig. 5, however, is that for the SCP the amount of single ionization during the second half cycle is more than double the amount produced by a single HCP. We interpret the additional single ionization as indicating the contribution of rescattering events.

Figure 6 shows the time dependence of double ionization for both the SCP and the DHP. Here again, one sees that 


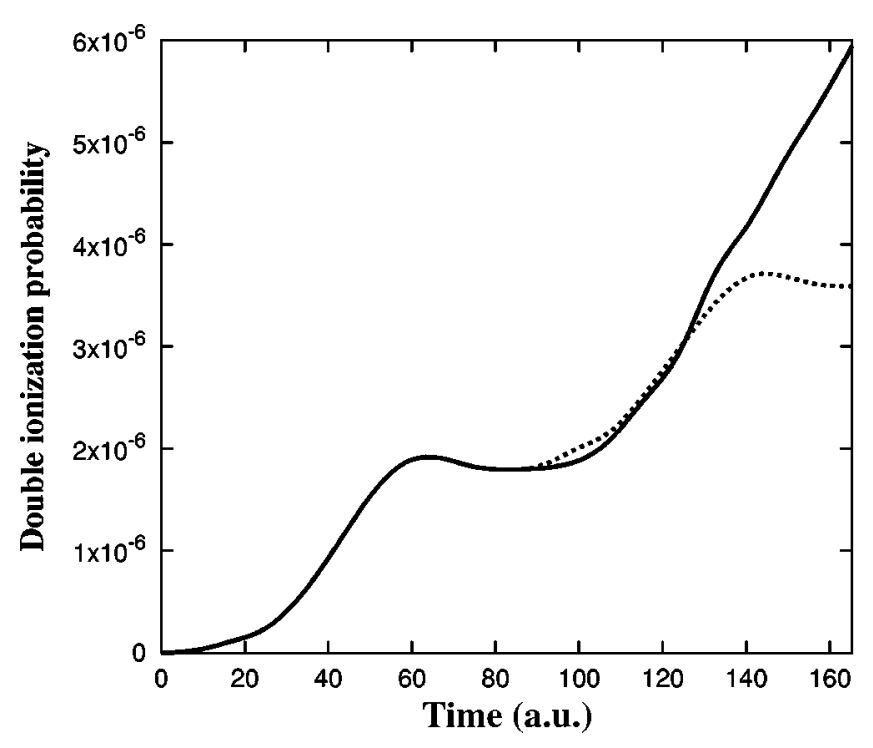

FIG. 6. Double-ionization probability of $\mathrm{Li}^{-}$obtained using the single-cycle pulse (solid lines) and the double half cycle pulse (dashed lines). The parameters used for the two pulses are the same as in Fig. 5.

during the first half cycle, the double-ionization probability is the same for the two pulses, as expected, but that by the end of the second half cycle the double-ionization probability for the SCP is significantly larger than that for the DHP. We see here that the second HCP in the DHP produces roughly the same double ionization as is produced by the first HCP; the absence of interference between the two wave packets produced may be interpreted classically as stemming from the fact that the dominant configuration for double ionization is for the two electrons to leave in opposite directions, so that the Coulomb repulsion between them hastens their departure from the interaction region before the onset of the second HCP. The key point of Fig. 6 is that double ionization is also enhanced by the recollision that occurs during the second half cycle for the SCP. This is a signature of correlated nonsequential double-ionization, because if the two electrons were ionized sequentially and independently, the two pulses would in principle yield the same double ionization probability. The enhancement observed for the SCP during the second half cycle is due to the recollision, which increases electron-electron and electron-core interactions. In fact, during this recollision, one can envision the following possibilities: (i) The returning electron wave packet may acquire more energy in its recollision with the ion core in order to be definitely ionized, thereby leading to a larger singleionization probability for the SCP than the DHP, as discussed above. (ii) The returning electron may ionize the other electron in an $e-2 e$ collision process assisted by the field, leading also to an enhancement of double-ionization for the SCP. It is worth noting that, as shown in Fig. 6, there is a sharp increase in the double ionization probability during the last quarter of the SCP. This is consistent with our classical calculations in Sec. II, which indicate that rescattering electrons that are born in the continuum with a nonzero velocity (which is the case here, since we are in the above-barrier regime) return later in the second half cycle (see Fig. 2). The angular distributions for double ionization, discussed in the following section, provide additional insights into the contributing mechanisms for the double-ionization process.

\section{ANGULAR DISTRIBUTIONS FOR DOUBLE IONIZATION}

We have recently developed a technique for obtaining angular distributions for double ionization by ultrashort, intense laser pulses $[12,13]$. The resulting doubly differential double-ionization probability (DDDIP) is not differential in energy; it therefore accounts for all possible energy transfers to electrons from the field pulse, as well as for all possible energy-sharing configurations between the two electrons. The DDDIP is doubly differential in the solid angles $d \Omega_{j}$ $=\sin d \theta_{j} d \phi_{j}(j=1,2)$, and yields the energy-integrated double-ionization probability for any given combination of the four spherical angles $\theta_{1}, \phi_{1}, \theta_{2}, \phi_{2}$ of the two electrons. [The position vector of each electron is $\mathbf{r}_{j}$ $\equiv\left(r_{j}, \theta_{j}, \phi_{j}\right)$.] More details on the calculation of the DDDIP are given in Refs. [12,13].

As the DDDIP depends on four spherical angles, one needs to fix two of these angles to be able to make threedimensional (3D) plots of the double-ionization probability results as a function of the other two angles. In presenting the results, we make two choices for the azimuthal angles, $\left(\phi_{1}=0, \phi_{2}=0\right)$ and $\left(\phi_{1}=0, \phi_{2}=\pi\right)$, which both correspond to the case of coplanar emission of the two electrons. For the coplanar case, these two choices provide complete information about the ejection directions of the two electrons, because of the symmetry of the system with respect to the polarization axis $\hat{z}$. The laser polarization axis divides the emission plane in question into two half planes. In the case $\left(\phi_{1}=0, \phi_{2}=0\right)$, a plot of the DDDIP with respect to the polar angles $\theta_{1}\left(0 \leqslant \theta_{1} \leqslant \pi\right)$ and $\theta_{2}\left(0 \leqslant \theta_{2} \leqslant \pi\right)$ corresponds to double ejection of the two electrons in the same half plane. The case $\left(\phi_{1}=0, \phi_{2}=\pi\right)$ corresponds to double ejection of the two electrons in opposite half planes (i.e., electron 1 ejected in one half plane and electron 2 in the other half plane). Note finally that our angular distribution results are presented only at the end of the electric-field pulse, for the cases of a HCP, a SCP, and a DHP. Detailed comparisons of the results for these three cases follow.

The DDDIPs obtained at the end of a HCP, which is equal to the first half cycle of both the SCP and the DHP shown in Fig. 4, are plotted in Fig. 7(a) for the case $\left(\phi_{1}=0, \phi_{2}=0\right)$, and in Fig. 7(b) for the case $\left(\phi_{1}=0, \phi_{2}=\pi\right)$. Globally, Fig. 7 indicates that the double-ionization probability is mostly concentrated along the polarization axis in the regions corresponding to $\theta_{1} \approx \pi$ and/or $\theta_{2} \approx \pi$. In other words, at least one of the two electrons is ejected at a small angle with respect to the negative direction of the polarization axis, and for this reason there is little difference between the two cases shown in Figs. 7(a) and 7(b). This agrees with the fact that during the first half cycle, the force due to the field accelerates electrons in this direction. A more detailed analysis of Fig. 7 shows additional interesting features.

(i) The double-ionization probability is negligible for twoelectron ejection in the region $\left(\theta_{1} \approx 0, \theta_{2} \approx 0\right)$, which corre- 

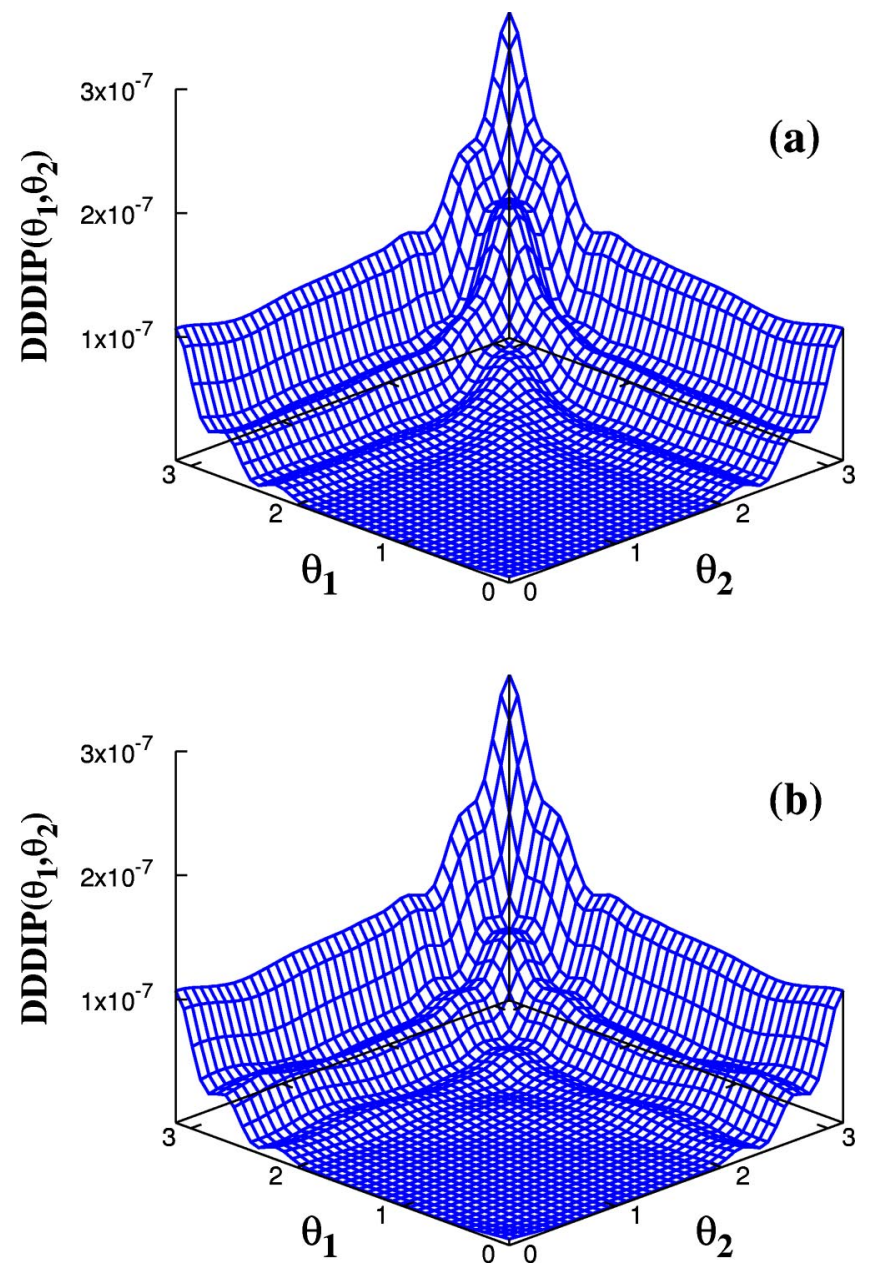

FIG. 7. Coplanar doubly differential double-ionization probability (DDDIP) for $\mathrm{Li}^{-}$at the end of a half cycle pulse (which equals the first half cycle of both the single-cycle pulse and the double half cycle pulse), as a function of the polar angles $\theta_{1}$ and $\theta_{2}$ (in radians) of the two electrons: (a) for $\phi_{1}=\phi_{2}=0$, and (b) for $\phi_{1}=0$ and $\phi_{2}=\pi$. The parameters used for the two laser pulses are the same as in Fig. 5.

sponds to the positive direction of the polarization axis. In other words, the ejection of both electrons in the direction opposite to the field force is negligible, as expected.

(ii) A broad peak in the DDDIP is located in the vicinity of ( $\theta_{1} \approx \pi, \theta_{2} \approx \pi$ ), corresponding to the ejection of both electrons at zero or at small relative angles in the negative direction along the polarization axis. This result is also expected, because this is the direction in which the field kicks electrons during the first half cycle. This is also an indication that sequential double ionization dominates. Previous calculations with more conventional light pulses have found this double ejection configuration $[12,13,33]$.

(iii) Finally, there is a significant probability for twoelectron ejection in the vicinity of $\left(\theta_{1} \approx 0, \theta_{2} \approx \pi\right)$ or equivalently, in the vicinity of $\left(\theta_{1} \approx \pi, \theta_{2} \approx 0\right)$, which indicates the ejection of one electron along the direction of the field force and the other in the opposite direction.

Figure 8 presents, in spherical coordinates, threedimensional plots of the DDDIP for one electron when the
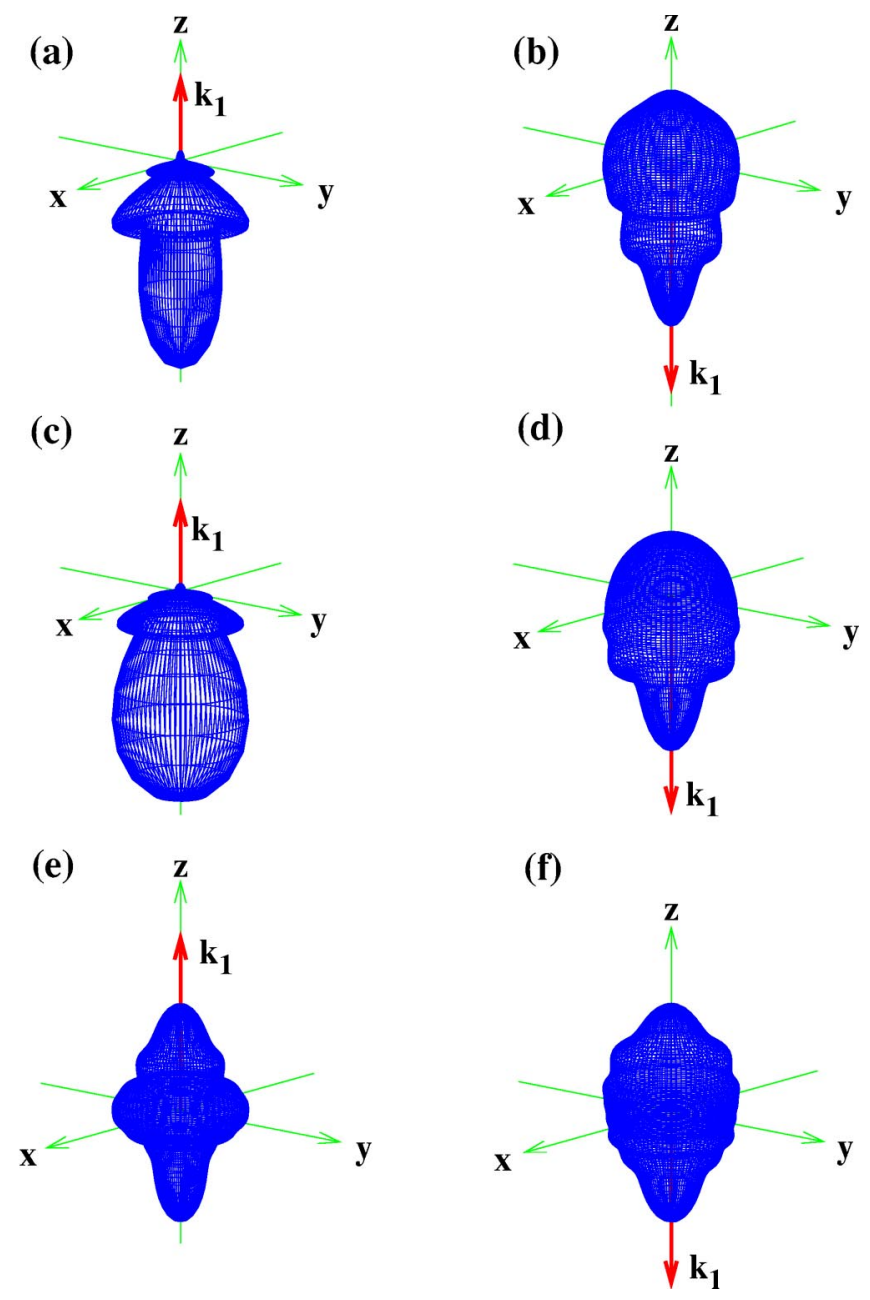

FIG. 8. DDDIP for $\mathrm{Li}^{-}$in spherical coordinates. In each case, the plot shows the angular distribution of one electron (say, electron 2 ), when the other (electron 1 ) is ejected in the direction given by the unit vector $\mathbf{k}_{1}$ along the polarization axis $z$, which is an axis of symmetry: (a) and (b) are obtained at the end of a half cycle pulse, which equals the first half cycle of both the DHP and the SCP; (c) and (d) are obtained at the end of the DHP; and (e) and (f) are obtained at the end of the SCP. The parameters used for the pulses are the same as in Fig. 5.

other is ejected in a fixed direction given by the unit vector $\mathbf{k}_{1}$ along the polarization axis $\hat{z}$. The DDDIP obtained at the end of the HCP is shown in Fig. 8(a) for the case in which electron 1 is ejected in the positive direction along the $\hat{z}$ axis (i.e., $\theta_{1}=0$ ), and in Fig. 8(b) for the case in which electron 1 is ejected in the negative direction along the $\hat{z}$ axis (i.e., $\theta_{1}=\pi$ ). It appears from Fig. 8(a) that if electron 1 is ejected in the positive direction along $z$, the probability for ejection of electron 2 in the same direction is negligible [which corresponds to the case (i) discussed in the previous paragraph]; electron 2 appears predominantly in the opposite direction. In contrast, Fig. 8(b) shows that if electron 1 is ejected in the negative direction along $z$, there is a strong probability for electron 2 to be ejected in the same direction at zero or small relative angles due to the field force [cf. the lobe below the $x y$ plane in Fig. 8(b)]. However, there is also a significant 
probability for electron 2 to be ejected in the direction opposite to that of electron 1 [cf. the lobe above the $x y$ plane in Fig. 8(b)].

Two-electron ejection in opposite directions along the $\hat{z}$ axis, shown in Figs. 8(a) and 8(b) after the HCP, provides clear evidence of ejection mechanisms other than solely the direct action of the field. This result cannot be explained by a sequential uncorrelated double-ionization mechanism; needless to say, it also cannot be explained by any recollision mechanism since the field force remains in the same direction during the $\mathrm{HCP}$, so that there is no force driving the electron back to the parent ion. Rather, this is a clear signature of a nonsequential double ionization that is mediated by electron-electron correlations, which forces one electron to appear in the continuum in the direction opposite to that of the other electron, or at a large relative angle with respect to the other. The only mechanisms possibly leading to such angular distributions are those in which one electron is ejected by the action of the field and the other via Coulomb electron-electron and core-electron interactions. Such mechanisms are the shake-off, the knockout, and possibly multiphoton-sharing ones. During the HCP (or, equivalently, during the first half cycle of either the SCP or the DHP), as the field intensity increases sharply, one electron is ejected following absorption of one or many photons, the other electron is shaken off, as it cannot adiabatically adjust to the new ionic potential (shake-off). Also, after absorbing one or more photons, one electron may eject the other on its way out (knockout). Finally, if the first electron absorbs sufficient energy to be above the double-ionization threshold, it may share this energy with the other electron via correlation, leading to the ionization of both electrons (multiphoton sharing). In all cases, one electron appears predominantly along the polarization axis in the direction of the field force, while the other electron is ejected in the opposite direction or at a large relative angle with respect to the other, owing to electronelectron correlations. In fact, calculations of angular distributions for double ionization of He by a single high-energy photon have shown that in the shake-off, knockout, and photon-sharing mechanisms, the two electrons emerge predominantly at large relative angles [2]. More precisely, the two electrons emerge predominantly in opposite directions for the shake-off and photon-sharing mechanisms, and at $90^{\circ}$ relative angles for the knockout mechanism [2]. Unfortunately, it is neither possible in our calculations to isolate the contributions of any one of the three mechanisms mentioned above nor to quantify the contribution of each of them. However, it is clear that rescattering is to be ruled out for the double ionization that occurs during the HCP.

Plots of angular distributions for one electron when the other is ejected perpendicularly to the laser polarization axis are shown in Fig. 9. Results obtained at the end of the HCP [cf. Fig. 9(a)] indicate that if one electron is ejected perpendicular to the laser polarization axis, the other emerges predominantly along the $\hat{z}$ axis in the direction of the field force. It also appears that ejection of both electrons perpendicular to the field force has a small probability. Note that, for the configuration in which both electrons are ejected perpendicu- (a)

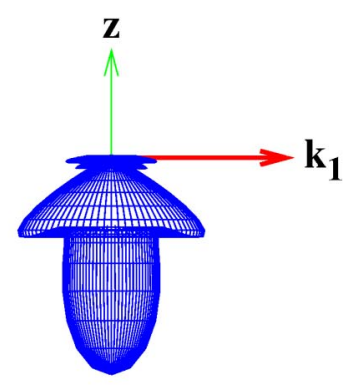

(b)

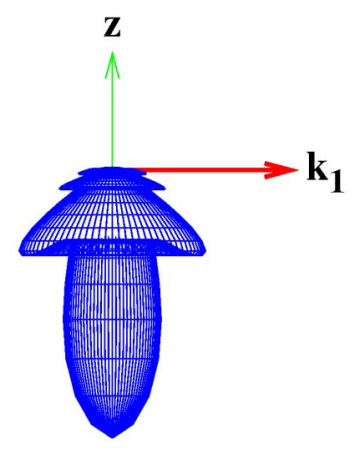

(c)

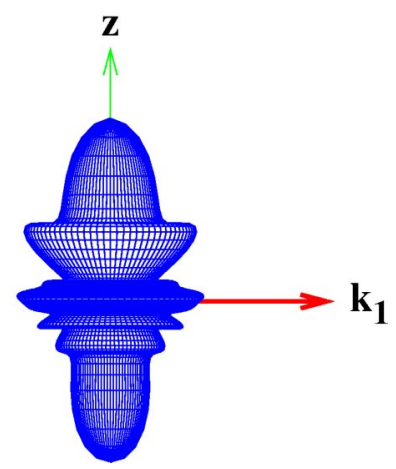

FIG. 9. DDDIP for $\mathrm{Li}^{-}$, in spherical coordinates, for one electron (say, electron 2), when the other (electron 1) is ejected perpendicular to the $\hat{z}$ axis along the unit vector $\mathbf{k}_{1}$ : (a) at the end of the HCP, (b) at the end of the DHP, and (c) at the end of the SCP. The parameters used for the pulses are the same as in Fig. 5.

larly to the $\hat{z}$-axis, the angular distributions obtained with wave-function components having a specific total angular momentum $L$ vanish for odd values of $L$ owing to very general symmetry considerations $[13,34]$.

The DDDIP obtained at the end of the DHP are shown in Fig. 10(a) for $\left(\phi_{1}=0, \phi_{2}=0\right)$ and in Fig. 10(b) for $\left(\phi_{1}\right.$ $\left.=0, \phi_{2}=\pi\right)$, and the corresponding three-dimensional spherical coordinate plots for two specific configurations are shown in Figs. 8(c) and 8(d). It appears that these DHP angular distributions are very similar in shape to those of the HCP in Figs. 7, 8(a), and 8(b). For the configuration in which one electron is ejected perpendicular to the $\hat{z}$ axis, the similarity is also evident from the comparison of angular distributions in Fig. 9(a) (obtained at the end of the HCP) and in Fig. 9(b) (obtained at the end of the DHP). This similarity is expected because for the DHP, what happens during the sec- 

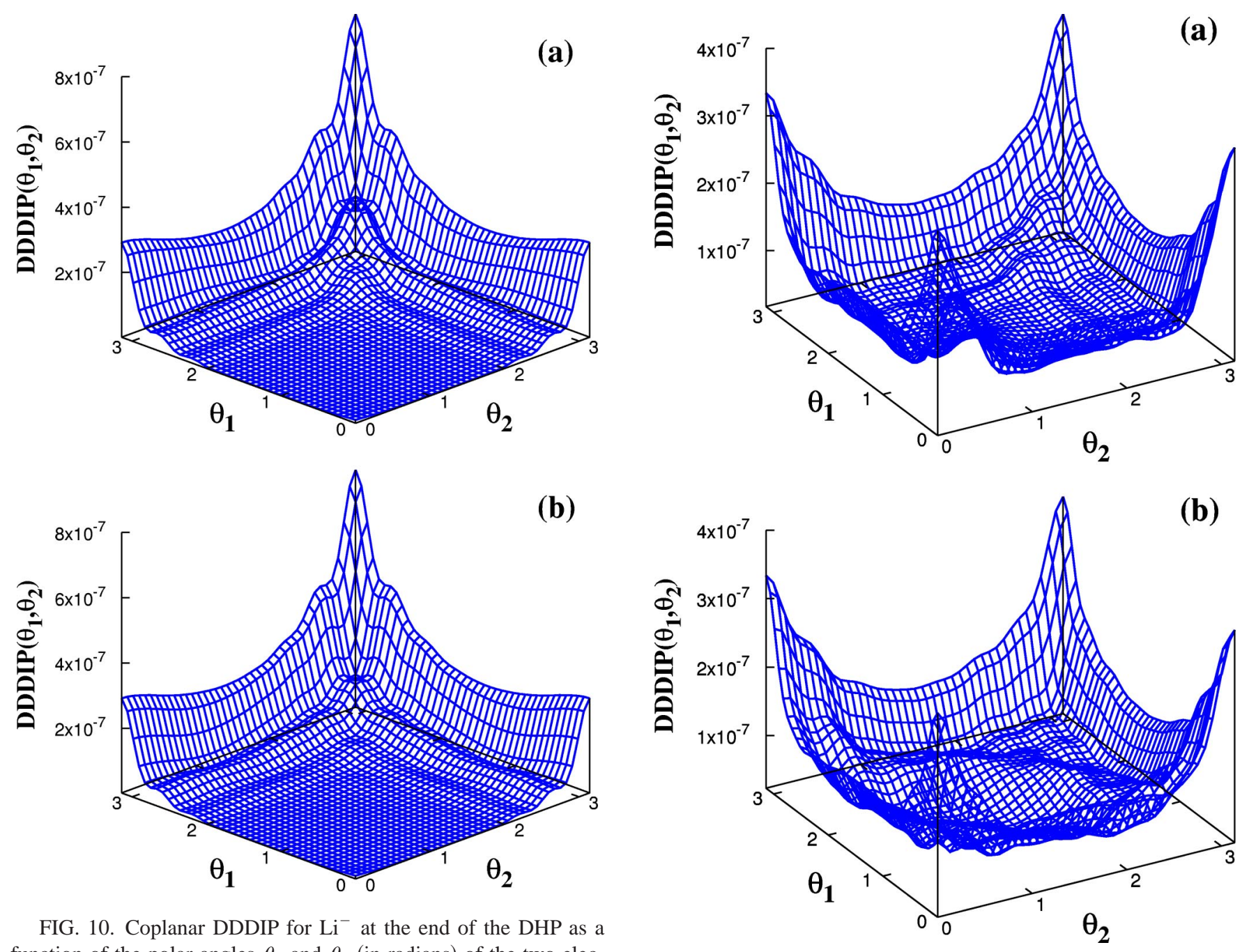

FIG. 10. Coplanar DDDIP for $\mathrm{Li}^{-}$at the end of the DHP as a function of the polar angles $\theta_{1}$ and $\theta_{2}$ (in radians) of the two electrons: (a) for $\phi_{1}=\phi_{2}=0$, and (b) for $\phi_{1}=0$ and $\phi_{2}=\pi$. The parameters used for the two pulses are the same as in Fig. 5.

ond half cycle is very similar to what occurs during the first one: electrons receive a second kick in the same direction. However, the increase in magnitude of the angular distribution indicates that the effects of the second half cycle add to those of the first half cycle. For this case, double ejection of both electrons at small relative angles in the same direction opposite to the field force is still negligible, while double ejection of the two electrons in opposite directions and in the same direction along the field force are significant.

For the SCP, the DDDIP is given in Fig. 11(a) for $\left(\phi_{1}\right.$ $\left.=0, \phi_{2}=0\right)$ and in Fig. 11(b) for $\left(\phi_{1}=0, \phi_{2}=\pi\right)$, and the corresponding spherical coordinate plots are shown in Figs. 8(e) and 8(f) for two specific configurations. The results also indicate that the double-ionization probability is dominant along the polarization axis. In particular, the plots in Fig. 11 show four prominent peaks corresponding to two-electron ejection in the four possible configurations along the $z$ axis: (i) both along positive $\mathbf{z}$ (cf. the peaks located in the vicinity of $\theta_{1}=\theta_{2}=0$ ), (ii) both along negative $\mathbf{z}$ (i.e., peaks located in the vicinity of $\theta_{1}=\theta_{2}=\pi$ ), (iii) electron 1 along positive $\mathbf{z}$ and electron 2 along negative $\mathbf{z}$ (i.e., peaks located in the vicinity of $\theta_{1}=0, \theta_{2}=\pi$ ); (iv) electron 2 along positive $\mathbf{z}$

FIG. 11. Coplanar DDDIP for $\mathrm{Li}^{-}$at the end of the SCP as a function of the polar angles $\theta_{1}$ and $\theta_{2}$ (in radians) of the two electrons: (a) for $\phi_{1}=\phi_{2}=0$, and (b) for $\phi_{1}=0$ and $\phi_{2}=\pi$. The parameters used for the two pulses are the same as in Fig. 5.

and electron 1 along negative $\mathbf{z}$ (i.e., peaks located in the vicinity of $\theta_{1}=\pi, \theta_{2}=0$ ). Note that the configuration (i), originally negligible in Figs. 7 and 10, is prominent in Fig. 11 , thereby illustrating the effects of the change in direction of the field force for the SCP. Also, for the configurations in which one electron is ejected along the laser polarization axis, cylindrical symmetry implies that it makes little difference in which half plane the other electron is ejected so that Figs. 11(a) and 11(b) are similar.

Another consequence of the rescattering is that the double-ionization probability at large angles with respect to the polarization axis, in particular, in the directions perpendicular to the polarization axis, are now substantial [cf. the region corresponding to $\theta_{1}$ and $\theta_{2}$ both close to $\pi / 2$ in Fig. 11(b)]. Figure 9(c) shows, in 3D spherical coordinates, the distribution of electron 2 when electron 1 is ejected along $\mathbf{k}_{1}$, perpendicular to the $\hat{z}$ axis. Comparing Fig. 9(c) with Figs. 9(a) and 9(b), one sees that in contrast to the HCP and DHP cases, double ejection of both electrons perpendicular to the laser polarization axis is substantial for the SCP. Due 
to the electron-electron repulsion, the DDDIP in Fig. 11(b), which corresponds to two-electron ejection perpendicular to the $\hat{z}$ axis in opposite half planes, is slightly larger than its counterpart in Fig. 11(a) for double ejection perpendicular to the $z$ axis in the same half plane. This signature of electronelectron repulsion is more pronounced in Fig. 11 than in Figs. 7 and 10 because of the recollision that enhances electron-electron interactions for the SCP.

\section{CONCLUSIONS}

For the multiphoton and above-barrier ionization regimes considered in this paper, tunneling is negligible, and the ionized electron most likely appears in the continuum with a nonzero velocity, in contrast to the zero velocity that is typical of the tunneling regime. Our solutions of the classical equations of motion for a single electron born in the continuum with nonzero velocity in the laser field (and without accounting for its interaction with the atomic core) indicate that the electron may nevertheless return to the origin for a possible recollision with the core. This means that rescattering is a potential contributing mechanism for double ionization in the multiphoton or above-barrier regime. However, the return of the electron to the origin depends on the time at which it is ejected in the continuum, and, most importantly, on the parameter $\beta=\sqrt{K_{0} / 2 U_{p}}$, where $K_{0}$ is the initial kinetic energy of the electron and $U_{p}$ is the ponderomotive potential. With increasing $\beta$, the electron returns to the atomic core with increasingly less kinetic energy, and for $\beta$ larger than the classical cutoff $\beta_{c} \approx 1.2172$, the electron does not return at all (according to the classical model).

We have also performed full-dimensional calculations (including two-electron correlations) for $\mathrm{Li}^{-}$, treated as a twoactive electron system, interacting with an intense SCP and a DHP, for parameters corresponding to the multiphoton above-barrier regime. Besides the signature of the dominating sequential double ionization, our numerical investigations have uncovered signatures of the shake-off, the knock- out, and the multiphoton-sharing double-ionization mechanisms, in addition to evidence for the rescattering mechanism. The single- and double-ionization yields are found to be significantly larger for the SCP than for the DHP, which clearly illustrates the influence of the rescattering mechanism that occurs only for the SCP. Angular distributions for double ionization obtained at the end of a HCP (which equals the first half cycle of both the SCP and the DHP), during which the field force acts in only one direction, shows electron ejection in the direction opposite to the field force, i.e., in the double ionization by a HCP, one electron may appear in the direction of the field force and the other in the opposite direction. This is a clear signature of nonsequential double ionization mediated by electron-electron correlations. The possible mechanisms leading to such double ejection are those for which one electron is ejected by the action of the field and the other by electron-electron and/or electron-core interactions. Such mechanisms are the shakeoff, the knockout, and possibly multiphoton-sharing ones. Therefore, for a laser field in the multiphoton above-barrier regime, these are potentially contributing mechanisms for double ionization.

Finally, our numerical "experiments" have elucidated the physical mechanism for ejection of both electrons perpendicular to the laser polarization axis, which was first predicted in our calculations employing short, many cycle pulses $[12,13]$. We have found here that these configurations occur with much higher probabilities for a single-cycle pulse than for a half cycle or a double half cycle pulse. These configurations thus appear to be signatures of the electron recollision mechanism of double ionization.

\section{ACKNOWLEDGMENTS}

This work was supported in part by the U.S. Department of Energy, Office of Basic Energy Sciences, Chemical Sciences, Geosciences, and Biosciences Division, under Grant No. DE-FG03-96ER14646.
[1] J.S. Briggs and V. Schmidt, J. Phys. B 33, R1 (2000).

[2] Z.J. Teng and R. Shakeshaft, Phys. Rev. A 47, R3487 (1993); 49, 3597 (1994).

[3] J.H. McGuire, N. Berrah, R.J. Bartlett, J.A.R. Samson, J.A. Tanis, C.L. Cocke, and A.S. Schlachter, J. Phys. B 28, 913 (1995).

[4] A. Kheifets, J. Phys. B 34, L247 (2001).

[5] D.N. Fittinghoff, P.R. Bolton, B.C. Chang, and K.C. Kulander, Phys. Rev. Lett. 69, 2642 (1992).

[6] B. Walker, B. Sheehy, L.F. DiMauro, P. Agostini, K.J. Schafer, and K.C. Kulander, Phys. Rev. Lett. 73, 1227 (1994).

[7] Th. Weber, M. Weckenbrock, A. Staudte, L. Spielberger, O. Jagutzki, V. Mergel, F. Afaneh, G. Urbasch, M. Vollmer, H. Giessen, and R. Dörner, Phys. Rev. Lett. 84, 443 (2000).

[8] R. Moshammer, B. Feuerstein, W. Schmitt, A. Dorn, C.D. Schröter, J. Ullrich, H. Rottke, C. Trump, M. Wittmann, G. Korn, K. Hoffmann, and W. Sandner, Phys. Rev. Lett. 84, 447 (2000).
[9] K.J. Schafer, B. Yang, L.F. DiMauro, and K.C. Kulander, Phys. Rev. Lett. 70, 1599 (1993); P.B. Corkum, ibid. 71, 1994 (1993).

[10] B.A. Zon, JETP 89, 219 (1999); U. Eichmann, M. Dörr, H. Maeda, W. Becker, and W. Sandner, Phys. Rev. Lett. 84, 3550 (2000).

[11] X. Tang, H. Rudolph, and P. Lambropoulos, Phys. Rev. A 44, R6994 (1991); C.A. Nicolaides, S. Dionissopoulou, and T. Mercouris, J. Phys. B 29, 231 (1996); J. Parker, K.T. Taylor, C.W. Clark, and S. Blodgett-Ford, ibid. 29, L33 (1996); M.S. Pindzola and F. Robicheaux, Phys. Rev. A 57, 318 (1998); A. Scrinzi and B. Piraux, ibid. 58, 1310 (1998); G. Lagmago Kamta, Th. Grosges, B. Piraux, R. Hasbani, E. Cormier, and H. Bachau, J. Phys. B 34, 857 (2001).

[12] G. Lagmago Kamta and A.F. Starace, Phys. Rev. Lett. 86, 5687 (2001).

[13] G. Lagmago Kamta and A.F. Starace, Phys. Rev. A 65, 053418 (2002). 
[14] B. Feuerstein, R. Moshammer, and J. Ullrich, J. Phys. B 33, L823 (2000).

[15] V.R. Bhardwaj, S.A. Aseyev, M. Mehendale, G.L. Yudin, D.M. Villeneuve, D.M. Rayner, M.Yu. Ivanov, and P.B. Corkum, Phys. Rev. Lett. 86, 3522 (2001).

[16] S.V. Popruzhenko and S.P. Goreslavskii, J. Phys. B 34, L239 (2001).

[17] M. Dörr, in Multiphoton Processes, edited by L.F. DiMauro, R.R. Freeman, and K.C. Kulander (AIP, Melville, NY, 2000), p. 176.

[18] M. Lein, E.K.U. Gross, and V. Engel, Phys. Rev. Lett. 85, 4707 (2000).

[19] A. Becker and F.H.M. Faisal, Phys. Rev. A 59, R1742 (1999); Phys. Rev. Lett. 84, 3546 (2000).

[20] R. Kopold, W. Becker, H. Rottke, and W. Sandner, Phys. Rev. Lett. 85, 3781 (2001).

[21] J. Andruszkow et al., Phys. Rev. Lett. 85, 3825 (2000).

[22] See, e.g., G.G. Paulus, W. Becker, W. Nucklich, and H. Walter, J. Phys. B 27, L703 (1994), and references therein.

[23] M. Hentschel, R. Keinberger, Ch. Spielman, G.A. Reider, N. Miloscevic, T. Brabec, P. Corkum, U. Heizmann, M. Drescher, and F. Krausz, Nature (London) 414, 509 (2001).

[24] T. Brabec and T. Krausz, Rev. Mod. Phys. 72, 545 (2000).

[25] B.I. Greene, J.F. Federici, D.R. Dykaar, R.R. Jones, and P.H. Bucksbaum, Appl. Phys. Lett. 59, 893 (1991); D. You, R.R. Jones, D.R. Dykaar, and P.H. Bucksbaum, Opt. Lett. 18, 290 (1993).

[26] T.F. Gallagher, Phys. Rev. Lett. 61, 2304 (1988)
[27] J.L. Krause, K.J. Schafer, and K.C. Kulander, Phys. Rev. Lett. 68, 3535 (1992).

[28] C. Leubner and P. Zoller, J. Phys. B 13, 3613 (1980).

[29] W.E. Lamb, Jr., R.R. Schlicher, and M.O. Scully, Phys. Rev. A 36, 2763 (1987).

[30] L.B. Madsen, Phys. Rev. A 65, 053417 (2002).

[31] The terms "laser," "frequency," and "peak intensity" are in quotes because unlike the SCP, the DHP is not a conventional laser pulse. One may argue that this is also the case for terms such as "multiphoton," etc. However, we extend these terms to the DHP by analogy with the SCP case. We remove the quotes in subsequent references to these quantities.

[32] Back of the envelope estimates confirm the possibility of significant intererence between the two wave packets. If one assumes that wave packet 1 is created at the peak of the first half cycle by absorption of a single photon, then wave packet 1 has a kinetic energy of 0.016 a.u. (i.e., a velocity of 0.18 a.u.). Thus there is only a time delay of a quarter of a laser period (41.3 a.u.) before wave packet 2 begins to be produced. During this time delay, the wave packet 1 would travel a distance of only 7.3 a. u., which is less than the radius of the ground-state wave function, indicating that the second wave packet would likely interfere with the first wave packet (i.e., this will be a kind of Ramsey interference).

[33] M.G. Makris, L.A.A. Nikolopoulos, and P. Lambropoulos, Europhys. Lett. 54, 772 (2001).

[34] G. Lagmago Kamta and Anthony F. Starace, J. Mod. Opt. 50, 597 (2003). 